\title{
Características epidemiológicas de los pacientes con paraparesia espástica tropical (PET) asociada a infección por HTLV-1 en Lima, Perú.
}

\author{
Epidemiological aspects in patients with tropical spastic paraparesis associated to HTLV-1 \\ infection in Lima, Peru.
}
De las Casas César ${ }^{1}$, Gotuzzo Eduardo ${ }^{1},{ }^{2}$, Deza Luis ${ }^{3}$, Cabrera Juan $^{2}$, Castañeda César ${ }^{3}$, Watts Douglas 4 .

${ }^{1}$ Instituto de Medicina Tropical Alexander Von Humboldt. Universidad Peruana Cayetano Heredia. Lima, Perú.

${ }^{2}$ Hospital Nacional Cayetano Heredia. Lima, Perú.

${ }^{3}$ Hospital Nacional Guillermo Almenara Irigoyen (IPSS). Lima, Perú.

${ }^{4}$ NAMRID. Lima, Perú.

\section{RESUMEN}

Objetivo: Describir las características epidemiológicas de la paraparesia espástica tropical asociada a infección por HTLV-1. Material y métodos: Se realizó un estudio descriptivo de 61 pacientes consecutivos con paraparesia espástica tropical (PET) asociada a HTLV1. Los casos fueron evaluados prospectivamente en dos instituciones de Lima (Instituto de Medicina Tropical "Alexander Von Humboldt" y el Servicio de Neurología del Hospital Nacional Guillermo Almenara Irigoyen) entre agosto de 1989 y noviembre de 1995. Fueron incluidos 61 pacientes con diagnóstico clínico de PET y confirmación sexológica de HTLV-1 mediante ELISA y Western Blot. Resultados y conclusiones: Se observó una preponderancia del sexo femenino, con una relación mujer a hombre de 1.91:1. La edad promedio fue de 51.7 años (rango: 21 a 76 años). El 50.5\% había residido en regiones andinas, siendo éste el lugar de nacimiento para el $54.1 \%$. Sólo el $6.6 \%$ fue referido desde hospitales de esa región. No se identificaron adictos a drogas endovenosas, aunque un 23\% tenía antecedente de transfusiones. Enfermedades de transmisión sexual fueron admitidas por el 35\% de los hombres que respondieron, en los que la falta de empleo del condón fue un evento frecuente. Otros factores de riesgo fueron también evaluados. (Rev Med Hered 1996; 7: 68-74).

PALABRAS CLAVE: HTLV-1, paraparesia espástica tropical (PET), epidemiología, Perú. 


\section{SUMMARY}

Objective: To define the epidemiological aspects of tropical spastic paraparesis associated with HTLV-1. Material and methods: This is a descriptive study of 61 consecutive patients with tropical spastic paraparesis (TSP) associated with HTLV-1 infection in Peru seen at two reference centers in Lima between august 1989 and November 1995. All patients had serologic evidence of HTLV-1 infection measured with reactive ELISA test and confirmed positive Western Blot assays. Results and conclusions: The female-male ratio was 1.91:1. The mean age at the time of diagnosis was 51.70 years (range: 21 to 76 years). A considerable proportion of patients (70.5\%) had lived in Andean regions, basically due to their place of birth (54.1\%). Only $6.6 \%$ of cases were referred from hospital of these regions, there were no intravenous drug addicts (IVDA) although 23.0\% admitted transfusions. Sexually transmitted diseases were reported by $35 \%$ of males, in which lack of condom use was a common finding. Other possible risk factors also were studies. (Rev Med Hered 1996; 7: 68-74).

KEY WORDS: HTLV-1, tropical spastic paraparesis (TSP), epidemiology, Perú.

\section{INTRODUCCIÓN}

Desde 1911, en que Rous (1) descubre la transmisibilidad del sarcoma de pollo a partir de un "agente filtrante" (un virus), se han identificado otros virus que producen tumores sólidos y leucemia en animales. La mayoría de estos virus oncogénicos fueron clasificados en una sola familia: Retroviridae. Sin embargo, el descubrimiento de la existencia de un retrovirus humano tuvo que esperar hasta 1980, cuando Poiesz y col. reportaron el aislamiento de un retrovirus tipo C en un paciente que padecía una malignidad de células T considerada una variante agresiva de micosis fungo idees y Síndrome de Sezary $(2,3)$. Actualmente conocemos a este virus como el "human T-cell lymphotropic virus (HTLV-1)".

En la cadena de transmisión del HTLV-1 múltiples mecanismos han sido reconocidos: madre a hijo (principalmente por lactancia materna) (6); sexual (tanto hetero como homosexual) $(2,7,8,9)$ y parenteral $(10,11)$ (transfusión sanguínea con componentes celulares y empleo de agujas contaminadas). Tal como sucede con otros retrovirus, la infección persiste de por vida, siendo el virus consistentemente aislado de personas seropositivas independientemente de su estado clínico (2).

El compromiso clínico de la infección por HTLV-1 se centra en dos entidades importantes: la Leucemia - Linfoma de células T del adulto (ATLL) y una paraparesia espástica progresiva también conocida como Paraparesia Espástica Tropical (PET) o Mielopatía asociada a HTLV-1 (HAM). Otros cuadros han sido ocasionalmente asociados a la infección por HTLV-1, pero la participación del virus en su etiopatogenia aún no está definida; entre ellos se ha descrito cuadros de polimiositis, alveolitos pulmonar, uveitis, Síndrome de Sjogren, artropatías, autoinfestación por Strongiloides, crioglobulinemia y gamapatías monoclonales, entre otros $(2,10)$.

En 1888 Strachan en Jamaica, describió a un grupo de pacientes que presentaban paraparesia con signos de piramidalismo, bajo la denominación de "neuritis múltiple de Jamaica" y atribuyó la 
etiología de la enfermedad a la malaria $(12,13)$. Unos 30 años más tarde. Scout, revisó los pacientes y las autopsias de Strachan y concluyó que en este grupo había varias enfermedades: una de ellas era una paraparesia con espasticidad $(12,13)$. En el Perú en 1952 se publica la tesis de Rodríguez quién describe un grupo de 24 pacientes del Hospital Santo Toribio de Mogrovejo con un Síndrome fundamental caracterizado por una paraplejia espástica con trastornos esfinterianos y concluye que la causa sería la sífilis, aún cuando no todos sus casos fueron comprobados (14). Posteriormente Montgomery, en 1969, acuñó el término de paraparesia espástica tropical (PET) para describir el síndrome neurológico que se observaba en algunos pacientes en Jamaica (12). La asociación entre PET y la infección por HTLV-1, fue demostrada por primera vez en Martinico en 1985 por Gessain y col. al observar que el 59\% de los pacientes con PET tenían anticuerpos para HTLV-1 (15). Pocos meses después, estudios en Jamaica y Colombia, detectaron anticuerpos para HTLV-1 en el suero y en el líquido cefalorraquídeo de pacientes on PER. A comienzos de 1986, Osame y col. describieron en Japón la asociación de este virus con una entidad clínicamente idéntica a PET, la cual designaron como "HTLV-1 associated myelopathy" (HAM) (16); se conoce desde entonces la causa del síndrome neurológico que había sido reconocido por más de un siglo por médicos de nuestro continente.

En Martinico, con una población total de 330,000 habitantes, se ha estimado que cerca de 10,000 individuos están infectados por el HTLV-1; el número de casos registrados de PET es próximo a 300, lo que representa el 3\% de las personas seropositivas. Una prevalencia similar de PET se ha registrado en Colombia. Si se asume una prevalencia mínima de $2 \%$ para aplicarse a lo largo del Caribe, Centro y Sud América, se puede estimar que existe alrededor de 100,000 casos de esta enfermedad paralítica progresiva, lo cual constituye un problema humano y de salud pública (4).

Al igual que el apoyo de laboratorio, el conocimiento de antecedentes epidemiológicos constituye un sustento importante para el diagnóstico de la PET asociado a HTLV-1 y para determinar las medidas de intervención con el fin de limitar la transmisión del agente.

La PET asociada a HTLV-1 fue descrita por primera vez en el Perú, por Jonson y col. en 1988 (18). Posteriormente, en el Instituto Nacional de Ciencias Neurológicas, Trilles describió casos similares (19). Estudios realizados en familiares de pacientes con PET en el mismo instituto han mostrado que el virus muestra agregación familiar y que un número importante de portadores provenían del trapecio andino (20).

El presente trabajo es un estudio colaborativo entre dos instituciones, cuyo objetivo es describir de manera prospectiva aspectos epidemiológicos en pacientes con PET e infección demostrada por HTLV-1.

\section{MATERIALES Y MÉTODOS}

El estudio incluyó 61 pacientes consecutivos con diagnóstico de PET e infección por HTLV-1 demostrada serológicamente atendidos entre agosto de 1989 y noviembre de 1995.

Los casos fueron recolectados de manera prospectiva y colaborativa en dos Instituciones de referencia con sede en Lima: el Instituto de Medicina Tropical "Alexander Von Humboldt" en el 
Hospital Nacional Cayetano Heredia y el servicio de Neurología del Hospital Nacional Guillermo Almenara Irigoyen.

Los criterios de Román y col. (21) fueron empleados con guía clínica, se determinaron como portadores de un cuadro clínico de PET a aquellos individuos que presentan debilidad de miembros inferiores asociados a espasticidad, acompañada con signos de liberación piramidal, disfunción esfinteriana y/o compromiso sensitivo. Otras causas de paraparesia tales como comprensión medular y enfermedades metabólicas o hereditarias fueron excluidas.

Se definió como infección por HTLV-1, cuando se demostraba la presencia sérica de anticuerpos contra el HTLV-1 mediante una reacción positiva contra la prueba de ELISA (Cambridge Bioscience, Worcester, Mass) y era confirmado por la presencia de las bandas p24 y gp46 con el test de Western Blot (DuPont, Wilmington, Del). Para proceder a la prueba de Western Blot, el ELISA debía ser reactivo en dos oportunidades. Las pruebas fueron realizadas en los laboratorios de Naval Medical Research Institute Datachment (NAMRID) en Lima.

\section{RESULTADOS}

Durante el periodo de estudio se identificaron 61 pacientes que cumplían con los criterios de inclusión. La proporción mujer a hombre fue 1. 91:1 (40 mujeres y 21 hombres), el grupo étnico más numeroso fue de los mestizos (78.7\%) y el resto lo conforman nativos andinos. La edad promedio del grupo fue de 51.7 años, con un rango entre 21 y 76 años. La edad promedio para los hombres fue 47.1 años y para las mujeres 54.2 años.

Los datos de región geográfica de nacimiento se resumen en la tabla $\mathrm{N}^{\circ} 1$. Una proporción importante de pacientes (52.5\%) han nacido en regiones andinas y muchos de los pacientes incluidos (70.5\%) han resistido por un tiempo importante en los Andes, sin embargo la mayoría de individuos (77\%) residían y provenían de Lima al momento de acudir a nuestras instituciones.

\begin{tabular}{|c|c|c|}
\hline \multicolumn{3}{|c|}{$\begin{array}{l}\text { Tabla N¹. Región geográfica de nacimiento de los } \\
\text { pacientes con PET asociada al virus HTLV-1. }\end{array}$} \\
\hline & $\mathrm{N}^{\prime}$ Pacientes & $\%$ \\
\hline Región Andina & 32 & 52.50 \\
\hline Costa del Pacífico * & 24 & 39.30 \\
\hline Selva Amazónica & 2 & 3.28 \\
\hline No Precisable & 3 & 4.92 \\
\hline TOTAL & 61 & 100.00 \\
\hline
\end{tabular}


Diversos posibles factores de riesgo reportados por los pacientes que dieron información precisa, se detallan a continuación (Tabla $\mathrm{N}^{\circ} 2$ ). El 24.6\% de los pacientes presentaban antecedentes de haber recibido transfusiones sanguíneas. En 11 de 14 casos, se conoce con certeza que la transfusión ocurrió antes de iniciarse la enfermedad; el intervalo promedio transcurrido entre este evento y el inicio de los síntomas fue de 10 años. Otros factores de riesgo incluyeron historia de acupuntura (13.2\%), el uso frecuente de inyectables intramusculares (67.3\%) y una historia previa de hepatitis (14.5\%). Sólo uno de los pacientes tenía tatuajes (1.9\%). No se encontraron adictos a drogas endovenosas. Dos pacientes reportaron antecedentes de linfoma en sus familiares.

\begin{tabular}{|c|c|c|c|c|}
\hline \multicolumn{5}{|c|}{ Tabla N². Evaluación de los posibles factores de riesgo en pacientes con PET } \\
\hline Factores & $\begin{array}{l}\text { Sobre tolal de pac. } \\
\text { con into. precisa }\end{array}$ & $\%$ & $\begin{array}{l}\text { Sobre tolal de } \\
\text { pac. estudiados }\end{array}$ & $\%$ \\
\hline Lactancia materna & $34 / 45$ & 75.6 & $34 / 61$ & 55.7 \\
\hline Inyecciones previas & $33 / 49$ & 63.7 & $33 / 61$ & 54.1 \\
\hline Cirugía previa & $27 / 46$ & 58.7 & $27 / 61$ & 44.3 \\
\hline RSP & $9 / 17$ & 52.9 & $9 / 61$ & 14.8 \\
\hline ETS en hombre & $7 / 20$ & 35.0 & $7 / 61$ & 11.5 \\
\hline Translusiones & $14 / 57$ & 24.6 & $14 / 61$ & 23.0 \\
\hline Hepatitis & $8 / 47$ & 14.5 & $8 / 61$ & 13.1 \\
\hline Acupuntura & 7153 & 13.2 & $7 / 61$ & 11.5 \\
\hline Drogas E.V. & $0 / 61$ & 0.0 & $0 / 61$ & 0.0 \\
\hline Drogas no E.V. & $2 / 56$ & 3.6 & $2 / 61$ & 3.3 \\
\hline RSH & $1 / 18$ & 5.6 & $1 / 61$ & 1.6 \\
\hline Tatuajes & $1 / 53$ & 1.9 & $1 / 61$ & 1.6 \\
\hline Prostitutas & $0 / 30$ & 0.0 & $0 / 61$ & 0.0 \\
\hline ETS en mujeres & $0 / 28$ & 0.0 & $0 / 61$ & 0.0 \\
\hline $\begin{array}{l}\text { RSP: Rolacionos soxualos } \\
\text { E.V.: Endovernosas; RSH: }\end{array}$ & $\begin{array}{l}\text { titutas: ETS: Entormedad of } \\
\text { s soxuales con homosexval }\end{array}$ & nsmis & & \\
\hline
\end{tabular}

Respecto a la evaluación de lactancia materna, el 75.6\% afirmó haber lactado durante su infancia y el 34.4\% restante respondió no saber con exactitud el hecho. En cuanto a conducta sexual, siete de veinte hombres (35\%) refirieron historia previa de enfermedades de transmisión sexual (ETS), nueve de diecisiete (52.9\%) admitieron relaciones con prostitutas y uno de dieciocho (56\%) relaciones homosexuales. El 91.6\% refirió no utilizar condón durante sus prácticas sexuales (once de quince individuos). La mayor parte de los hombres (73.3\%) negó haber tenido parejas sexuales en el último año. En las mujeres, en la información sobre su conducta sexual, ninguna refirió antecedentes de prostitución o ETS: dos pacientes refirieron antecedentes de "úlcera genital". 


\section{DISCUSIÓN}

El HTLV-1 fue el primer retrovirus en ser identificado y relacionado en algún tipo de cáncer. Durante la última década diversos estudios han mostrado que la infección por HTLV-1 es endémica en algunas áreas del mundo, siendo las dos áreas mejores estudiadas las islas del suroeste del Japón (4) (donde aproximadamente 16\% de la población adulta es seropositiva) y las Islas del Caribe (5) (en donde 2-5\% de los adultos negros son seropositivos). Otras áreas endémicas incluyen regiones de Centro y Sud América (Colombia, Venezuela, Perú), África Ecuatorial y las Seychelles (2,4). En los Estados Unidos, la infección por HTLV-1 ha sido identificada principalmente en adictos a drogas endovenosas (e.v) y en inmigrantes de áreas endémicas.

En nuestro país ha sido reconocida una alta tasa de infección en el grupo de meretrices, como fue observado en estudios realizados en Lima (7) y en el Callao (22) (prevalencias de 7\% y 25\% respectivamente). Asimismo, estudios epidemiológicos realizados en Cuzco y Quillabamba han identificado a esta zona como una región endémica: la tasa global encontrada fue 5.1\% y llegaba hasta $13.7 \%$ en el grupo de prostitutas (23). En una población de pacientes con SIDA adquirido por vía sexual, se ha identificado al HTLV-1 (en infecciones duales con VIH) en el 18\%; la progresión del SIDA en estos pacientes fue más agresiva y mostró una mortalidad más temprana $(8,24)$.

En 1988, se identificaron en el Perú los primeros casos de PET asociados a HTLV-1 (18). Sin embargo, el cuadro neurológico era confrontado por nuestros médicos desde casi un siglo atrás y su causa atribuida a la malaria, la sífilis y/o intoxicaciones $(14,19)$.

En la actualidad, es preciso determinar la distribución del HTLV-1 en nuestro territorio, los factores de riesgo asociados con su transmisión y su comportamiento clínico, para poder diseñar medidas de intervención.

Las variables demográficas estudiadas muestran concordancia con lo descrito en la literatura. Así, la predominancia del sexo femenino en casos de PET en nuestro estudio, con una relación 1.91:1, ha sido descrita en Chile (2.2:1), Japón (1.5:1), Martinica (3.5:1) y Colombia (1.5:1). Este hecho puede reflejar diferencias ligadas al sexo en la eficiencia de la transmisión del virus $(12,15,25,26)$. Estudios en Japón muestran que la transmisión hombre-mujer es de $61 \%$ en una pareja estable para un período de 10 años, mientras que la transmisión mujer-hombre en 10 años es menor de $1 \%(27,28)$.

La edad promedio en nuestra serie fue de 51.7 años al momento del diagnóstico ( $\mathrm{SD}=12.01)$ y se calculó en 46.9 años al momento del inicio de la enfermedad ( $\mathrm{SD}=12.9)$. Estos datos concuerdan con otras series de la literatura $(12,15,25,26)$.

Una proporción importante de pacientes han nacido (54.1\%) y/o han residido (70.5\%) en regiones andinas, aunque al momento de acudir a nuestras instituciones la mayoría residía en Lima, esto puede sugerir que la exposición a factores de riesgo para adquirir la infección por HTLV-1 ocurre en alguna fase temprana de la vida en áreas endémicas. Esto ha sido observado 
también en estudios en poblaciones que migran de áreas endémicas como son los japonesesamericanos que viven en Hawai, en estas poblaciones, la elevada prevalencia de infección por HTLV-1 se encuentro asociada al lugar de nacimiento (10).

$\mathrm{Al}$ analizar factores de riesgo, se evidenció que once pacientes (19.3\%) tenían una historia de transfusión sanguínea previa al inicio de los síntomas. Datos similares han sido recogidos de pacientes con PET en Japón y en Martinico, donde la prevalencia oscila entre 13\% y 20\% (29).

La transmisión del virus ocurre sólo con la transfusión de productos sanguíneos con componentes celulares (sangre total, paquete globular, concentrado de plaquetas). No se han reportado casos en pacientes hemofílicos que recibieron sólo factores de coagulación (2). La tasa de seroconversión en pacientes transfundidos con componentes expuestos al HTLV-1 fue de 63\% en Japón y 44\% en Jamaica (30). Estos hallazgos señalan diferencias en los mecanismos de transmisión del HTLV-1 con respectivo al VIH. Es importante notar también, que el inicio de la enfermedad en los casos asociados a transmisión vía transfusión sanguínea es relativamente corto. Casos de PET asociados a transfusión han ocurrido desde pocos meses después del evento.

La media entre el episodio transfusional y el inicio de síntomas en nuestro estudio fue de 12.3 años. Se ha determinado que los pacientes con mayor riesgo de seroconversión son aquellos que reciben sangre seropositiva almacenada menos de una semana, los receptores de múltiples transfusiones y aquellos inmunosuprimidos al momento de la transfusión. Es interesante resaltar que solamente casos de PET han sido consistentemente asociados a transfusiones sanguíneas y no así casos de ATLL (31).

El uso de agujas contaminadas ha sido incriminado en la transmisión de varios retrovirus en poblaciones de adictos a drogas endovenosas $(32,33)$. Si bien ésta no es una práctica frecuente en nuestro país (ninguno de nuestros pacientes dio historia de abuso de drogas endovenosas), el uso de agujas y jeringas para medicación parenteral sin medidas mínimas de bioseguridad es frecuente y puede resultar un vehículo efectivo para la transmisión del agente. Hipótesis similares han sido planteadas por investigadores en otros países (33). Es importante resaltar que un número importante de nuestros pacientes dio historia de uso frecuente de inyectables (66\%), aunque una valoración más adecuada de esta cifra requiere compararla con un grupo control.

El estudio seroepidemiológico de Cuzco y Quillabamba reveló que el uso frecuente de inyectables y los procedimientos dentales se asociaron en forma significativa a una mayor seropositividad en la población adulta sana (23). La historia de intervenciones quirúrgicas es alta en nuestro grupo de pacientes (58.7\%). Estudio en Chile, mostraron una proporción similar de pacientes con historia previa de intervenciones quirúrgicas, aunque el análisis estadístico no mostró significancia para este parámetro (20).

Acupuntura y tatuajes son otros mecanismos de transmisión horizontal de otro virus, como hepatitis B y VIH. Hemos encontrado el antecedente de acupuntura en una proporción importante de pacientes (13.2\%), pero no así el dato de tatuajes (1.9\%). Sin embargo, nuestra encuesta no precisa el momento exacto en el cual se realizó la acupuntura, pudiendo este hecho corresponder a que los pacientes con PET presentan en muchos casos dolor lumbar que los lleva a buscar distintas terapias frente al dolor, como la acupuntura. 
Nuestro estudio describe el antecedente de hepatitis en el 14.55\% de los casos registrados.

El estudio de Cuzco y Quillabamba asocia como factor de riesgo la historia de ictericia (23); considerando que zonas de selva alta y trapecio andino son endémicas para fiebre amarilla y hepatitis B y delta, esta asociación podría sugerir un mecanismo de transmisión compartido por estos virus y HTLV-1.

La lactancia materna es una vía reconocida de transmisión vertical del virus (2). Se ha observado que infantes que no lactan muestran menores tasas de seroconversión que aquellos que sí lo hacer (34). En el suroeste del Japón, el 20-30\% de los infantes que lactaron de madres infectadas, se infectaron durante el primer año de vida, mientras que sólo el 1-2\% de los alimentados con fórmulas mostraron seroconversión (6). La leche de las madres seropositivas ha mostrado un alto número de linfocitos infectados por el virus los cuales serían los vehículos de transmisión (35).

La abstinencia de la lactancia materna podría romper el ciclo de endemicidad en algunas poblaciones $(2,35)$. En nuestro estudio, el antecedente de lactancia materna es reconocido por el $75.6 \%$ de los pacientes, pero se requiere de un grupo control, pues la lactancia materna es una práctica frecuente en la población general.

Se ha visto que los pacientes con PET no tienen un riesgo incrementado de desarrollar linfoma comparado con la población general de portadores de HTLV-1 (2). La agregación familiar de la infección por este virus hace posible que familiares de pacientes con PET que sean portadores, puedan desarrollar ATLL o Mielopatía (2). En nuestro estudio se identificaron dos pacientes con familiares con antecedentes de linfoma.

La transmisión sexual del virus HTLV-1 hombre-mujer, hombre-hombre y raramente mujerhombre, es bien reconocida en la literatura $(2,37)$. La pareja receptiva es más susceptible que la contraparte receptiva es más susceptible que la contraparte insertiva, probablemente reflejando la ineficiente transmisión de este retrovirus altamente dependiente de células. Para las mujeres, el riesgo de infección muestra correlación con el número de parejas sexuales durante su vida, así como con los títulos de anticuerpos contra HTLV-1 que presentan sus parejas (10).

En un estudio cerca de homosexuales en Trinidad, se observó que la seroprevalencia para VIH fue tres veces mayor que la de HTLV-1 (40\% vs. 15\%), lo que sugiere que el HTLV-1 es transmitido menos eficiente por contacto homosexual que el VIH (38). En nuestro estudio sólo uno de diecisiete (5.9\%) pacientes reconoció haber tenido contacto homosexual, nueve de diecisiete (52.9\%) afirmaron relaciones sexuales con prostitutas y un número importante de pacientes hombres refirió antecedentes de enfermedad de transmisión sexual (35\%).

Es importante destacar que el 91.6\% de pacientes señaló que nunca empleaba condón durante sus prácticas sexuales. El virus ha sido detectado consistentemente en células mononucleares del semen de hombres seropositivos (2), lo que a la para de la baja tasa de transmisión de mujer a hombre sugiere que el semen es un vehículo importante para la transmisión sexual del virus y que el uso de condones podría prevenir la transmisión sexual del virus. El estudio de infección por HTLV-1 en prostitutas de Lima destaca la asociación entre la duración de la prostitución, la 
falta de uso consistente del condón y una historia de infección por Chlamydia trachomatis con una seropositividad significativamente alta al HTLV-1 entre las prostitutas estudiadas (7).

$\mathrm{Al}$ analizar el antecedente de enfermedad de transmisión sexual en mujeres, sólo dos pacientes en nuestra serie señalaron historia de úlcera genital; sin embargo en un porcentaje importante de pacientes mujeres de dato no fue recolectable. Ninguna de las mujeres evaluadas afirmó haber practicado la prostitución.

El 66.6\% de las pacientes no emplearon métodos anticonceptivos, 22\% empleó anticoncepción hormonal y 11\% refirió el uso de dispositivos intrauterinos. Se reconoce que el empleo de anticonceptivos orales aumenta la prevalencia de ectopía cervical asociada a la edad y que la ectopía cervical puede aumentar el riesgo de adquirir el VIH. Dos estudios prospectivos en Nairobi encontraron asociación entre el uso de anticonceptivos orales y el riesgo de infección por VIH $(39,40)$. Sin embargo, la relación entre ectopía cervical e infección por HTLV-1 requiere investigaciones prospectivas.

La PET ha sido conocida desde por lo menos un siglo en el Perú. La prevención será siempre el mejor tratamiento para las enfermedades asociadas a retrovirus. A pesar que no existe a la fecha vacuna o cura contra el HTLV-1, existe suficiente información para lograr el control de esta infección.

En nuestro país necesitamos precisar mejor las áreas endémicas, así como el de instituir un programa de despistaje para HTLV-1 en los productos sanguíneos celulares de los bancos de sangre, investigar los factores de riesgo en las regiones del trapecio andino y educar a la comunidad general y médica respecto a este problema de salud pública.

\section{REFERENCIAS BIBLIOGRÁFICAS}

1.Rous P. A sarcoma of the fowl transmissible by an agent separable from the tumor cells. J Exp med 1911; 13: 397-411.

2.Stoeckle M. Introduction-Type C oncoviruses including Human T-Cell Leukemia Virus Types I an II. In: Mandell G, Douglas G, Bennett J, eds. Principles and Practice of Infectious Diseases $3^{\text {rd }}$. ed. New York: Churchill Livingstone, 1990.

3.Poeisz BJ. Ruscett FW, Gazdar AF, et al. Detection and isolation of type C retrovirus particles from fresh and cultures lymphocytes of a patients with cutaneous T-cell lymphoma. Proc Natl Acad Sci USA 1980; 77: 7415-7419.

4.Montgomery RD. HTLV-1 and tropical spastic paraparesis 1. Clinical features, pathology and epidemiology. Trans Roy Soc Trop Med Hyg 1989; 83: 724-728.

5.De-The G. Gessain A, Gazzolo L, et al. Comparative seroepidemiology of HTLV-1 and HTLV-111 in the French West Indies and some African countries. Cancer Res 1985; 45: Suppl: 4633s-4636s.

6.Ando Y, Nakano S, Satio K, et al. Transmission of adult T-cell leukemia retrovirus (HTLV-1) from mother to child: Comparison of bottle - fed with breast-fed, Jpn J Cancer Res 1987; 78: 322-324.

7.Gotuzzo E, Sánchez J, Escamilla J, et al. Human T cell lymphotropic virus type I infection among female sex workers in Perú. J Infect Dis 1994; 169: 754-759. 
8.Gotuzzo E, Escamilla J, Phillips IA, et al. Human T cell lymphotropic virus type I/II infection on the prognosis of sexually acquired cases of acquired inmunodeficiency syndrome. Arch Intern Med 1992; 152: 1429-1432.

9.Gessain A, Gout O. Chronic Myelopathy associated with human T-lymphotropic virus type I (HTLV-1). Ann Inter Med 1992; 117: 933-946.

10.Yanagihara R. Human T-cell lymphotropic virus I Infection and Disease in the Pacific basin. Human Biology 1992; 64: 843-854.

11.Rezza G, Titti F, Rossi G, et al. Sex as a risk factor for HTLV-1 spread among IV drug abusers. Lancet 1988: I: 713.

12.Zaninovic V. Paraparesia espástica tropical en Colombia. En: Enfermedad asociadas con el virus HTLV-1. Bogotá, Colombia. 1988 .p. 77-86.

13.Strachan H. On a form of multiples neuritis prevalent in the West Indies. Practitioner 1988; 59: 477.

14.Rodríguez M. Paraplejia espástica sifilítica de Erb. Tesis de Bachiller. Facultad de Medicina Universidad Nacional Mayor de San Marcos. 1952.

15.Gessain A, Vernant JC, Mauro L. et al. Antibodies to human T-Lymphotropic virus type I in patients with tropical spastic paraparesis. Lancet 1985; 2: 407-409.

16. Osame M, Usuku K, Izumu S, et al. HTLV-1 associated myelopathy, a new clinical entity. Lancet 1986; 11: 1031.

17.De The G, Bomford R. An HTLV-1 Vaccine: Why, How for whom?. AIDS Res Hum Retroviruses 1993; 9: 381-386.

18.Johnson RT, Griffin DE, Arregui A, et al. Spastic paraparesis and HTLV-1 infection in Perú. Ann Neurol 1988; 23(suppl): 151-155.

19.Trelles L, Tropical spastic paraparesis in Perú. En HTLV-1 and the nervous system. Alan-R. Liss, Inc 1989; 157-165.

20.Rodríguez JJ. Detección de portadores del virus HTLV-1 en las familias de pacientes con PET o HAM en el Perú. Tesis para optar el Título de Médico Cirujano, Universidad Peruana Cayetano Heredia, 1992.

21.Roman GC, Spencer PS, Schoenberg BS. Tropical myeloneuropathies: the hidden endemias. Neurology 1985; 35: 1158-1170.

22.Wignall FS, Phillips IA, Hyams KD, et al. Sexual transmission of human T-lymphotropic type I in peruvian prostitutes. J Med Virol 1992; 38: 44-48.

23.Zurita S, Costa C, Gotuzzo E, y col. Prevalencia de infección retroviral en diferentes grupos poblacionales de las ciudades de Quillabamba y Cuzco, Perú. Presentado en el VI Congreso Panamericano de Infectología 26-29 de mayo de 1993. Resumen 219.

24.Schechter M. Harrison L, Halsey N, et al. Coinfection with human T-cell lymphotropic virus HTLV-1 and HIV in Brazil. JAMA 1994; 271: 353-357.

25.Cartier L, Araya F, Castillo JL. et al. Progressive spastic paraparesis associated with human tcell leukemia virus type (HTLV-1). Intern Med 1992; 31: 1257-1261.

26.Vernant JC, Marus L, Gout O, et al. Associated tropical spastic paraparesis in Martinique: a reappraisal. Ann Neurol 1988: 23S: 133-135.

27.Stuver S, Tachibana N, Okayama A, et al. Heterosexual transmission of human $\mathrm{T}$ cell leukemia/lymphoma virus type I among married couples in southwestern Japan: An initial report from the Miyazaki Cohort Study. J Infect Dis 1993; 167: 57-65.

28.Kajiyama W, Kashiwagi S, Ikematsu H, et al. Intrafamilial transmission of adult T leukemia virus. J Infect Dis 1986; 154: 851-857. 
29. Osame M, Janssen R, Kubota H, et al. Nationwide survey of HTLV-1 associated myelopathy in Japan: association with blood transfusion. Ann Neurol 1990; 28: 50-56.

30.Chorba TL, Jason JM, Ramsey RB, et al. HTLV-1 antibody status in hemophilia patients treated with factor concentrates prepared from U.S. plasma sources, and in hemophilia patients with AIDS. Thromb Haemost 1985; 53: 180-182.

31. Ocochi K, Sato H, Hinuma Y. A retrospective study on transmission of adult T cell leukemia virus by blood transfusion: seroconversion in recipients. Vox Sang 1984; 46: 245-253.

32.Arango C, Concha M, Zaninovic V, et al. Epidemiology of tropical spastic paraparesis in Colombia and associated HTLV-1 infection. Ann Neurol 1988; 23S: 161-16.

33.Williams AE, Fang CT, et al. Human T-lymphotropic virus type 1 screening in volunteer blood donors - United States, MMWR 1989; 39: 915-921-923.

34.Kinoshita K, Amagasaki T, Hino S, et al. Milk-borne transmission of HTLV-1 from carrier mothers to their children. Jpn J Cancer Res 1987; 78: 674-680.

35.Hino S. Milk-borne transmission of HTLV-1 as a manor route in the endemic cycle. Acta Paediatr 1989; 21: 428-435.

36.Furukawa Y, Fujisawa J, Osame M, et al. Frequent clonal proliferation of human T-cell in HTLV-1. Associated Myelopathy (HAM-TSP), Blood 1992; 80: 1012-1016.

37.Blattner WA. Epidemiology of HTLV-1 and associated diseases. In Blattner WA, ed. Human retrovirology: HTLV New York: Raven Press, 1990.

38.Bartolomew C, Saxinger WC, Clark JW, et al. Transmission of HTLV-1 and HIV among homosexual men in Trinidad. JAMA 1987; 257: 2605-2608.

39.Moss GB, Clementson D, D'Costa L, et al. Association of cervical ectopy with heterosexual transmission of human immunodeficiency virus; results of a study of couples in Nairobi, Kenya. J Infect Dis 1991; 194: 588-591.

40.Plummer FA, Simonsen JN, Cameron DW, et al. Cofactors in male-female sexual transmission of human immunodeficiency type I. J Infect Dis 1991; 164: 588-591.

\section{Correspondencia}

Dr. Eduardo Gotuzzo H.

Instituto de Medicina Tropical "Alexander Von Humboldt".

Honorio Delgado s/n

San Martín de Porres.

Telf. 482-3410/482-3910 